\section{Targeting the core of resistance}

ARE-1
blockade of
the ARE might
be a way to
overcome
several
mechanisms of
enzalutamide
resistance

The sequence preference of the glucocorticoid receptor (GR) ligand binding domain (LBD) is similar to that of the androgen receptor (AR) and signalling through the GR results in gene expression that overlaps with that induced by AR signalling. Hence, the GR can be co-opted to overcome enzalutamide-induced AR blockade. A new study shows that a pyrrole-imidazole polyamide called ARE- 1 , which binds the androgen response element (ARE), is active in enzalutamide-resistant prostate cancer cells, suggesting it might help overcome resistance to AR LBD antagonists.

Pyrrole-imidazole polyamides can bind to DNA with sequence specificity and high affinity resulting in disruption of transcription factor binding and downstream events. Kurmis et al. first showed that ARE-1 reduces proliferation of LNCaP/AR cells, which are castration resistant through $\mathrm{AR}$ overexpression. In $\mathrm{VCaP}$ cells, which have modest GR expression in addition to AR overexpression resulting in reduced enzalutamide activity, and enzalutamide-resistant LREX' cells, ARE-1 treatment reduced proliferation, regardless of induction of AR or GR signalling with dihydrotestosterone or dexamethasone, respectively.

Using RNA sequencing and gene set enrichment analysis, the team showed that dihydrotestosterone treatment of LREX' cells resulted in enrichment of the AR signalling pathway, which was abrogated by ARE-1. In addition, the UV DNA damage response pathway was enriched in these cells. UV radiation causes damage to the DNA structure that results in arrest of RNA polymerase II and degradation of its subunit RPB1. Levels of nascent RNA in these cells were reduced, suggesting that ARE-1 treatment can reduce global transcription levels.

Finally, the researchers tested the efficacy of ARE-1 in VCaP and LREX' mouse xenograft models. In castrated LREX' models, addition of ARE-1 to enzalutamide treatment resulted in $80 \%$ reduced tumour growth compared with enzalutamide only. In addition, these tumours had reduced KLK3 RNA expression, increased DNA fragmentation via TUNEL assay and reduced Ki67 staining. RNA sequencing showed induction of a UV response signature similar to the in vitro setting.

ARE-1 blockade of the ARE might be a way to overcome several mechanisms of enzalutamide resistance, owing to its interference downstream of the signalling cascade.

\section{Clemens Thoma}

ORIGINAL ARTICLE Kurmis, A. A. et al. A pyrrole-imidazole polyamide is active against enzalutamide-resistant prostate cancer. Cancer Res. http://dx.doi.org/10.1158/0008-5472.CAN16-2503 (2017)

FURTHER READING Narayanan, S. et al.

Androgen-glucocorticoid interactions in the era of novel prostate cancer therapy. Nat. Rev. Urol. 13, 47-60 (2016) 